\title{
La investigación en ecología química en México
}

\author{
ANA LUISA ANAYA LANG \\ Instituto de Fisiología Celular, UNAM. Apdo. Postal 70-243. 04510, México, D.F.
}

\begin{abstract}
Resumen. La ecología química es una ciencia que surgió formalmente en los años 50's. Uno de los trabajos pioneros en ecología química se realizó en México. Lincoln Brower y colaboradores, en los años 60, realizaron los estudios, hoy clásicos, sobre ecología evolutiva de plantas tóxicas y sus herbívoros asociados. En 1989 comienza a tomar mayor impulso la ecología química realizada en México. En el presente trabajo se lleva a cabo un recorrido por los principales aspectos de la ecología química que han sido estudiados en nuestro país, con un énfasis especial en los diversos aspectos de la alelopatía. Además, se mencionan otros estudios sobre el papel de los metabolitos secundarios en la defensa de las plantas, la comunicación por medio de feromonas y la búsqueda de metabolitos con acción biológica diversa. La ecología química cobra un interés particular, debido a la potencial aplicación de los metabolitos secundarios como herbicidas, pesticidas, reguladores del crecimiento, antibióticos y agentes citotóxicos, entre otros. Es muy importante que los estudios de ecología química consideren la enorme biodiversidad de México, la cual representa al mismo tiempo una gran riqueza química. La exploración químico-ecológica puede formar parte de la conservación biológica, aunque existen también razones sociales y económicas que le dan mayor prioridad a la investigación sobre metabolitos secundarios activos
\end{abstract}

Abstract. Chemical ecology was formally born in the 50's. One of the pioneers' studies in chemical ecology was carried out in Mexico. Lincoln Brower and collaborators (in the 60'S) performed classic studies on evolutionary ecology of toxic plants and their associated herbivores. It was until 1989 that research on chemical ecology in Mexico began to take more impulse. Most of the main aspects of chemical ecology studied in Mexico are presented. Particular emphasis is made on the different aspects of allelopathy. Chemical ecology is more interesting because of potential application of secondary metabolites as herbicides, pesticides, growth regulators, antibiotics, and cytotoxic agents, among others. Chemical ecology studies should bear in mind the great Mexican biodiversity ranks fourth in the world, and hence representing a great chemical diversity. Therefore, chemical-ecological exploration could become part of biological conservation. Also, there are economic and social reasons that justify a research on active secondary metabolites.

\section{INTRODUCCIÓN}

La producción de diversos compuestos químicos por los organismos y su liberación al ambiente, afecta significativamente las condiciones de éste e influye sobre el crecimiento, la salud, la conducta y en suma la biología poblacional de plantas, animales y microorganismos, de modo distinto al relacionado con la nutrición; este hecho determina la existencia de interacciones bióticas particulares, las interacciones químicas entre los organismos.

Como es lógico, los esfuerzos coordinados de equipos de investigación formados por diversos especialistas, son las herramientas por excelencia en el difícil camino que debe recorrer este particular campo de la ciencia, siempre con el espíritu de observar los fenómenos, construir hipótesis, proyectos experimentales y proponer teorías, considerando a éstas como verdades parciales siempre sujetas a modificaciones y a rechazos, como en cualquier campo del quehacer científico.

Aunque algunos aspectos de lo que hoy conocemos como ecología química han sido motivo de estudio científico desde hace más de 50 años, particularmente en Europa, pero sin un marco de referencia conceptual formalizado. Como consecuencia, el desarrollo de esta ciencia fué muy lento en sus primeros 30 años. Sin embargo, algunas con- tribuciones científicas de primera línea establecen un verdadero parteaguas en el estudio y concepción de las interacciones ecológicas entre organismos, mediadas por las características químicas de los mismos.

1. En 1941, Müller y Börger estudiaron la reacción de diversas variedades de papa a cepas virulentas y avirulentas de Phytophtora. Las plantas sintetizaban compuestos de defensa ante este ataque. En 1960, Cruickshank y Perrin cristalizaron y caracterizaron químicamente la primera fitoalexina, la pisatina (Harborne, 1989).

2. Los estudios de Dethier (1954) y Fraenkel (1959) sugieren que los metabolitos secundarios de las plantas desempeñan un papel primordial como defensas contra los enemigos de éstas.

3. Los estudios de Ehrlich y Raven (1965) sobre ecología y evolución conjunta de mariposas y plantas, ponen de relieve la importancia de las interacciones químicas planta-animal.

4. Los estudios de Lincoln Brower y colaboradores sobre la ecología evolutiva de plantas productoras de cardenólidos y sus herbívoros asociados adaptados a estos metabolitos secundarios altamente tóxicos, en 
especial la mariposa monarca (Brower y Brower, 1964; Brower et al., 1968; Brower, 1969) constituyen uno de los más bellos y atractivos ejemplos en ecología química.

5. El trabajo de investigación sobre las interacciones químicas planta-planta y planta-microorganismo (aleloapatía) realizado en el Chaparral de California (Müller, 1970).

A la luz de estas investigaciones pioneras, nos empezamos a percatar de que el mundo natural, en relación con muchas interacciones ecológicas entre organismos, tiene una cierta estructura detectable y entendible, en la cual, hay una compleja serie de atributos químicos de los organismos, que hoy conocemos como infoquímicos, y que desempeñan un papel fundamental en la estructuración de la naturaleza, y al mismo tiempo han sido de gran importancia directa para el hombre (Dirzo y Anaya, 1989).

En los últimos 20 años, el avance de los métodos químicos que permiten el aislamiento, purificación y caracterización de productos naturales en microcantidades, y el interés de resolver ciertos problemas ecológicos urgentes, sobre todo aquellos relacionados con las pérdidas económicas causadas a la agricultura por plagas y malezas, permitió el florecimiento de esta rama de la ecología, a la vez rama de la química; un híbrido perfecto.

En México, hasta 1989, la ecología química empezaba apenas a ser perceptible en nuestro país, el cual resulta particularmente propicio para este tipo de estudios, dada su enorme riqueza biológica, así como por su enorme riqueza etnobiológica, es decir, la gran diversidad de modalidades que los distintos grupos étnicos, a través del tiempo, han utilizado para acoplarse y aprovechar los componentes de la exhuberante biodiversidad de esta región del planeta. A mayor riqueza biológica, mayor diversidad de interacciones biológicas (la mayoría de las cuales aun no hemos estudiado), las que a su vez conducen hacia la evolución de una mayor diversidad de productos naturales, muchos de los cuales aun no hemos descubierto, y cuyo potencial de utilidad para el hombre ni siquiera sospechamos (Dirzo y Anaya, 1989).

A continuación se mencionan algunas de las principales investigaciones hechas en México en diversos campos de la ecología química.

\section{Primeros estudios de alelopatía}

En 1970, se iniciaron los estudios sobre alelopatía en las zonas tropicales de México dentro de un proyecto sobre regeneración de selvas dirigido por el Dr. Arturo GómezPompa. Se investigó específicamente el potencial alelopático de algunas especies importantes de las primeras etapas de la sucesión secundaria en el área de la estación biólogica de la UNAM en Los Tuxtlas, Veracruz (Anaya, 1976; Anaya y Rovalo, 1976). Las especies seleccionadas con mayor potencial alelopático fueron: Piper auritum, P. hispidum, Croton pyramidalis y Siparuna nicaragüensis. Los aceites esencia- les de las Piperaceae fueron altamente inhibitorios sobre la germinación y el crecimiento de la mayoría de las plántulas de prueba. El $\alpha$ y $\beta$-pineno, dos constituyentes del aceite esencial de $P$. auritum , mostraron mayor actividad fitotóxica que el safrol, el cual constituye entre un $60 \%$ a $70 \%$ de este aceite (Collera, 1956; Rovalo, 1973; Anaya et al., 1987a).

Dentro del mismo proyecto, se realizó el estudio sobre el potencial alelopático de Ambrosia cumanensis, especie ruderal que crece vigorosamente y en poblaciones practicamente monoespecíficas (Anaya y del Amo, 1978). Las soluciones acuosas del suelo colectado bajo esta especie en julio (durante su floración) produjeron mayor inhibición sobre las especies de prueba que las del suelo colectado en diciembre. El estado fenológico y los cambios climáticos durante el año influyen sobre la cantidad y calidad de los metabolitos secundarios producidos y liberados al medio por las plantas. La descomposición de hojas y raíces de Ambrosia en el suelo de macetas también afectó a las especies de prueba. Este experimento que se realizó con suelo estéril y sin esterilizar puso en evidencia el papel que los microorganismos desempeñan en la liberación de diversos compuestos tóxicos, durante la descomposición de la materia orgánica. Las lactonas sesquiterpénicas producidas por A. cumanensis inhibieron o estimularon el crecimiento radical de las especies de prueba dependiendo de su concentración (del Amo y Anaya, 1978).

Nava-Rodríguez y colaboradores (1985) realizaron un estudio sobre el efecto de los aceites esenciales de estados juveniles de algunas especies primarias de Los Tuxtlas sobre el crecimiento de diversos hongos fitopatógenos, y pudieron observar que durante la época de lluvias se registró mayor daño por hongos sobre las hojas de las plantas, a la vez que aumentaban y se modificaban los metabolitos secundarios de los aceites esenciales, lo que puede ser interpretado como una respuesta de tipo fitoalexínico.

La información obtenida en esta parte del proyecto sobre Regeneración de Selvas en Los Tuxtlas, mostró que la alelopatía en el trópico puede contribuir a la eliminación de especies ya establecidas o a la selección de aquellas que empiezan apenas a establecerse dentro de la comunidad durante la sucesión secundaria, lo cual significa un control sobre la dirección que esta sucesión puede seguir (Anaya, 1976; Ramos-Prado, 1981).

\section{Estudios de alelopatía en agroecosistemas}

Gliessman y sus colaboradores del Colegio Superior de Agricultura Tropical en Cárdenas, Tabasco, llevaron a cabo diferentes estudios de alelopatía en agroecosistemas, especialmente en la región de la Chontalpa, entre 1979 y 1982. En este lugar, los campesinos clasifican a las malezas como «buen» o «mal monte». En general, el buen monte aparece tardíamente en los campos de cultivo porque estas plantas no pertenecen a las primeras etapas de la sucesión secundaria. Al contrario, el «mal monte» aparece tempranamente (Chacon y Gliessman, 1982). Durante las tareas de 
labranza, los campesinos eliminan al «mal monte» rápida y totalmente, y promueven a las otras arvenses por los múltiples beneficios que proporcionan.

La presencia de la maleza Cyperus rotundus en los agrosistemas puede reducir la incidencia de Tanatephorus cucumeris, un tizón del frijol (Phaseolus vulgaris). Por otro lado, Bidens pilosa puede controlar las poblaciones de diversos géneros de nemátodos parásitos de plantas que afectan severamente al maíz (Rosado-May et al., 1985, 1986).

Diversos estudios realizados en la zona de "camellones" (agrosistemas semejantes a las chinampas) en el suroeste del estado de Tlaxcala, demuestran que el arreglo de los policultivos permite el control de los insectos-plaga y el manejo de la fertilidad del suelo. Los árboles modifican el ambiente aéreo y las concentraciones de la mayoría de los nutrimentos en la superficie del suelo; éstas son mayores junto a los árboles y van disminuyendo a medida que la distancia aumenta. La abundancia de los artrópodos también varía, dependiendo de su grado de asociación con uno o más componentes del sistema (Altieri y Trujillo, 1987). Anaya y colaboradores (1987b) resaltan el papel de las plantas que funcionan como "trampa" para los insectos en los camellones, por ejemplo, Alnus sp. y Baccharis glutinosa. Durante el verano, ambas atraen a diversas plagas del maíz y otros cultivos, en especial al frailecillo Macrodactylus sp., evitando el ataque a los cultivos y la reducción de la productividad. También analizan las interacciones alelopáticas en un camellón que se sembró con maíz, frijol y calabaza, y donde se utilizaron algunas cubiertas vegetales (hojas de Alnus firmifolia, Betula erecta y Juncus $\mathrm{sp}$.). El número de malezas en las parcelas con estas cubiertas se redujo significativamente; la presencia y descomposición de las hojas modifica químicamente al suelo y afecta el crecimiento de las arvenses (Anaya, 1989).

El Programa de Agroecología de la Universidad de California en Santa Cruz y el Laboratorio de Ecología Química del Instituto de Fisiología Celular de la UNAM (Anaya et al., 1988) compararon los potenciales alelopáticos de siete de las principales arvenses comunes a Santa Cruz, California y a Texcoco, México: Chenopodium sp., Amaranthus sp., Raphanus raphanistrum, Brassica campestris, Sonchus oleraceus, Malva parviflora y Polygonum aviculare. Estas plantas se incorporaron al suelo de macetas y se observó su efecto sobre el crecimiento de malezas y maíz. Se constató que los factores como latitud, altitud y clima, afectan la producción de metabolitos secundarios en estas especies y por lo tanto su potencial alelopático. Las arvenses pueden ser útiles para la producción agrícola y las metas de conservación de recursos de los agricultores si son utilizadas de forma apropiada, interplantadas, o como cobertura o abono vegetal, dependiendo de la sensibilidad de los cultivos sobre los que se usen.

\section{Estudios de alelopatía en zonas áridas}

La dominancia y distribución espacial de Larrea divaricata en los desiertos mexicanos sugiere un mecanismo alelopático como elemento importante de las estrategias adaptativas de este arbusto. Los lixiviados acuosos de la parte aérea de la planta, colectada en los alrededores de Monterrey mostraron efectos inhibitorios selectivos sobre dicotiledóneas (Rovalo, 1978). En el caso de Helietta parvifolia (Rutaceae), especie del matorral submontano de Nuevo León, el potencial alelopático se acumula especialmente en las hojas, las cuales contienen alcaloides. Su aceite esencial muestra también propiedades fitotóxicas, bactericidas y fungicidas. Sin embargo, este aceite estimuló el crecimiento del frijol, demostrando que en la alelopatía el efecto sobre el crecimiento puede variar, dependiendo del tipo de compuestos que actúan o de la especie de organismo sobre el que actúan. El análisis de la vegetación reveló que $H$. parvifolia es la especie con mayor índice de importancia dentro de la comunidad. En la vecindad de esta planta existen numerosas plántulas de otras especies, sin embargo, nunca alcanzan su estado de madurez. Sin duda, algunos metabolitos secundarios liberados por $H$. parvifolia contribuyen a obstaculizar su desarrollo (Graue y Rovalo, 1982).

\section{Estudios químicos y fisiológicos en relación con la alelopatía}

González de la Parra y colaboradores (1981) identificaron dos monoterpenos diastereoisómeros, piquerol A y B de Piqueria trinervia, una maleza común en las zonas templadas y tropicales de México. Ambos compuestos mostraron un efecto fitotóxico selectivo, el piquerol A inhibió preferentemente el crecimiento de las raíces, y el piquerol $\mathrm{B}$ el de los tallos. Cruz-Ortega y colaboradores (1990) observaron que uno de los mecanismos de acción del diacetil-piquerol es la inhibición de la actividad de las $\mathrm{H}^{+}$-ATPasas de la membrana plasmática y de tonoplasto de raíces de Ipomoea purpurea. González de la Parra y colaboradores (1991) probaron la actividad del piquerol A y B sobre hembras de garrapata (Boophilus microplus), poniendo en evidencia su acción acaricida. La actividad de ambos piqueroles sobre este parásito es comparable a la actividad reportada de los acaricidas organofosfóricos.

El ácido fenil-acético es uno de los compuestos fitotóxicos del polen del maíz. Anaya y colaboradores (1992) comparan el efecto de pólenes de maíz y teosintle espolvoreados sobre semillas. El polen de teosintle no tiene ningun efecto sobre el crecimiento de las especies de prueba, en cambio el del maíz es altamente inhibitorio.

Diversas especies de la familia Brassicaceae son alelopáticas, por ejemplo, Brassica oleraceae y B. campestris. Las plantas de esta familia producen glucosinalatos que cuando se hidrolizan pueden formar isotiocianatos con propiedades tóxicas, aunque no autotóxicas. Los glucosinalatos inhiben por ejemplo, a Colletotrichum circinans, Botrytis sp., Sclerotium cepivorum y Aphanomyces euteiches. Por otro lado, afectan la actividad de las larvas de los nemátodos parásitos de raíces Meloidogyne incognita y Nacobbus aberrans (Zavaleta-Mejía, 1989). 
Castañeda y colaboradores (1992) aislan de un árbol endémico de la costa del Pacífico Mexicano, Celaenodendron mexicanum (Euphorbiaceae) algunos triterpenos (friedelina, maytensifolina B y el nuevo triterpeno 3b-hidroxifriedelan16-ona) que se reportan por vez primera como agentes alelopáticos y también flavonoides (ginkgetina, bilobetina y amentoflavona) los que igualmente mostraron efectos fitotóxicos.

La determinación de los mecanismos directos y los modos de acción por medio de los cuales los compuestos alelopáticos inhiben el crecimiento de las plantas, es la clave para encontrar algunos de los usos potenciales de los aleloquímicos. Al respecto, nuestro conocimiento es extremadamente escaso (Einhellig, 1989).

En algunas semillas las fitotoxinas con actividad autotóxica se encuentran frecuentemente en las partes externas, y si no son suficientemente lavadas por la lluvia o metabolizadas por la microflora del suelo, la semilla no germinará. Otras toxinas se encuentran en el interior de la semilla y son más difíciles de eliminar por lixiviación, como por ejemplo la cafeína del café y la estricnina de Strychnos nux-vomica (Waller et al., 1986, 1989). En este caso, las semillas deben tener mecanismos que les permitan evitar la autointoxicación. Durante la germinación, las semillas de café contienen un promedio de $40 \mathrm{mM}$ de cafeína; en condiciones experimentales, la mitosis de los meristemos radiculares de las plántulas de café se detiene con una concentración más baja, $10 \mathrm{mM}$. Esta concentración también provoca una inhibición casi total del crecimiento de la radícula y en gran medida del hipocotilo de las plántulas del café. En las semillas intactas, la cafeína se almacena en el endospermo mientras que el embrión permanece alejado de ella. Durante las primeras etapas de la germinación no se presenta división celular en los meristemos apicales de la radícula, hasta que la expansión y elongación celular empujan a ésta fuera de la semilla (1 a $3 \mathrm{~mm}$ fuera del endospermo), y por lo tanto, lejos de la cafeína. Así, la cafeína es conservada lejos de los sitios donde se lleva a cabo la división celular. La autotoxicidad de la cafeína se presenta también en la naturaleza (Anaya et al., 1982).

Calera y colaboradores (en prensa) realizaron un fraccionamiento biodirigido con el extracto metanólico de la raíz de Ratibida mexicana (Asteraceae) de donde se aislan dos lactonas sesquiterpénicas: la isoalloalantolactona y el elema-1,3,11-trien-8,12-olido. Ambos compuestos causan una inhibición significativa del crecimiento radical de Amaranthus hypochondriacus y Echinochloa crusgalli; además, ejercen una actividad citotóxica moderada contra tres líneas celulares tumorales e inhiben significativamente el crecimiento radial de tres hongos fitopatógenos. La isoalloalantolactona actúa inhibiendo la síntesis de ATP, la captura de protones y el transporte de electrones, actuando como un inhibidor de la reacción de Hill durante la fotosíntesis.

Uribe y colaboradores $(1984,1985)$ demostraron que uno de los compuestos activos del aceite esencial de Piper auritum, el B-pineno, desacopla el proceso de fosforilación oxidativa e inhibe la respiración en mitocondrias aisladas de levadura. Esta inhibición depende de la relación entre la concentración del terpeno y la cantidad de células de levadura; para una concentración fija de ß-pineno, la inhibición disminuye a medida que la cantidad de células de levadura aumenta. Estos efectos pueden ser explicados por la interacción del ß-pineno con la membrana mitocondrial.

Anaya y colaboradores (1990) estudiaron el potencial alelopático de Ipomoea tricolor, la cual es usada como abono verde en algunos cultivos con el fin de controlar malezas. Esta planta se usa además como purgante y sus semillas ricas en alcaloides se utilizan por sus propiedades alucinógenas en rituales mágicos. Se comprobó que en el principio purgante de esta planta, una resina glicosídica, radica principalmente el potencial alelopático de la misma. Posteriormente (Pereda-Miranda et al., 1993) identifican a la Tricolorina A como el componente mayoritario de esta resina y principal responsable de sus efectos fitotóxicos. Calera y colaboradores (en prensa) observan que esta resina inhibe la actividad de hidrólisis de ATP de la ATPasa de membrana plasmática. El porcentaje de inhibición fué de 25 a $35 \%$ en un rango de concentración de 20 a 200 mg. Por otro lado, la tricolorina A sola, inhibe la hidrólisis de ATP un poco más que la resina ( 21 a $38 \%$ ). La resina por ser hidrofóbica puede atravesar fácilmente la membrana.

\section{PRIMERA REUNIÓN NACIONAL DE ECOLOGÍA QUÍMICA EN MÉXICO}

La Primera Reunión Nacional de Ecología Química en México fue organizada en julio de 1989 por el Instituto de Fisiología Celular y el Centro de Ecología de la UNAM. Esta reunión puso en evidencia a diversas instituciones, grupos y personas dedicadas al estudio de esta disciplina y de otras estrechamente relacionadas con ella, por ejemplo del Instituto de Química y la Facultad de Química de la UNAM, el Centro de Investigaciones Ecológicas del Sureste, el Instituto de Ecología, el Colegio de Posgraduados, la Escuela Nacional de Ciencias Biológicas del Instituto Politécnico Nacional, La Universidad Autónoma Metropolitana y la Universidad Autónoma de Hidalgo.

Jiménez-Sierra y Dirzo (1989) comentaron que la diversidad vegetal en algunas comunidades puede ser explicada en parte, como el resultado de la influencia ejercida por los herbívoros. Estos autores, estudiaron algunos aspectos de la interacción planta-herbívoro en la Estación de Biología Tropical «Los Tuxtlas», de la UNAM, por medio de la observación de las preferencias alimenticias mostradas por un insecto herbívoro generalista (Melanonotus globosus), en condiciones de laboratorio. Se le ofrecieron a éste, 63 especies de plantas de la selva agrupadas de acuerdo a sus historias de vida en pioneras, nómadas y tolerantes. Los resultados de los experimentos de aceptabilidad se transformaron en índices con los que se hicieron comparaciones. El orden de preferencia en la aceptabilidad fue: pioneros $(0.21)$, nómadas $(0.10)$ y tolerantes (0.07). Sólo se apreciaron dife- 
rencias significativas entre los pioneros y los demás grupos. No hubo diferencia significativa entre la aceptabilidad a las hojas pubescentes y no pubescentes. La distribución de las características químicas y físicas, entre los grupos de especies arbóreas con distintas historias de vida, no fue homogénea, y concuerda en su mayoría con los postulados de la teoría general de la defensa óptima de los vegetales, y con la jerarquía de aceptabilidad media observada (se detectaron alcaloides en el $78 \%$ de las pioneras, el $64 \%$ de las nómadas y el $54 \%$ de las tolerantes).

Arízaga y Ezcurra (1989) observaron que el madroño es un árbol extremadamente variable en cuanto a las características foliares que presenta, pudiéndose encontrar formas desde piloso-glandulares hasta glabras. Al parecer, estos árboles forman un complejo de especies Arbutusxalapensis (Ericaceae) y probablemente, la característica de la pubescencia foliar es una respuesta ante la herbivoría. Diversas observaciones de campo han puesto en evidencia que los tricomas glandulares de los madroños glandulares atrapan insectos. Además, se ha encontrado una fuerte correlación entre la forma glandular de Arbutus xalapensis y los suelos pobres en nitrógeno, lo que hace suponer la posibilidad de utilización del nitrógeno protéico del insecto por parte del madroño. La forma glandular de Arbutus xalapensis está habitado preferentemente por insectos succionadores (Cyrtopeltis sp. Hemiptera: Miridae) con $76.7 \%$ del total, mientras que $15.1 \%$, corresponde a los insectos atrapados por los tricomas glandulares (Hymenoptera, fundamentalmente parasitoides, Coleoptera y Diptera). Para la forma glabra de Arbutus xalapensis, los insectos predominantes (53\%) son los succionadores, que forman agallas foliares (Tamalia $\mathrm{sp}$. Homoptera: Aphididae), y los masticadores (Lepidoptera) con $29 \%$ del total respectivamente. Por otra parte, la lámina foliar de la forma glabra del madroño, que es de mayor tamaño, es consumida en mayor grado por los herbívoros en comparación con las glandulares. Los metabolitos secundarios en ambos tipos de hojas de madroño, son similares, aunque las quinonas son más abundantes en las hojas glabras; otros aleloquímicos encontrados son flavonoides, glucósidos cardíacos y taninos.

La utilización de insecticidas sintéticos ha inducido el desarrollo de resistencia en muchas plagas de importancia agrícola, por ello, deben buscarse nuevos métodos de combate de insectos, acordes con la idiosincrasia del agricultor, económicos, de fácil aplicación y menos dañinos para el ambiente. Al respecto, nuestro país ofrece una gran alternativa por la enorme diversidad vegetal existente, la que significa mayor número de compuestos secundarios. Rodríguez-Hernández y Lagunes-Tejeda (1989) presentaron los resultados de 9 años de búsqueda de principios insecticidas en plantas; se han probado 437 especies de diferentes ecosistemas y se han descubierto 64 plantas tóxicas para el gusano cogollero del maíz Spodoptera frugiperda, siete para la conchuela del frijol Epilachna varivestis, ocho para el mosquito doméstico Culex quinquefasciatus, cinco contra el mosquito transmisor de la fiebre amarilla Aedes aegypti, 11 contra el gorgojo del maíz Sitophilus zeamais, 12 para el barrenador mayor de los granos Protephanus truncatus, 21 contra el gorgojo pardo del frijol Acanthoscelides obtectus y 6 contra el gorgojo pinto del frijol Zabrotes subfasciatus.

Villavicencio y Juárez-Monroy (1989) han desarrollado un bioensayo sistemático para evaluar diversos extractos de plantas como antialimentarios de Sitophilus granarius, insecto que ataca granos almacenados. Los extractos etanólicos de hojas frescas de: Thryallis glauca (Malpighiaceae), Decatropis bicolor (Rutaceae), Plumbago pulchella (Plumbaginaceae), P. capensis (Plumbaginaceae), Larrea tridentata (Zygophyllaceae), Rosmarinus officinale (Labiatae), Lippia graveolens (Verbenaceae), Calicarpasp.(Verbenaceae), Rumex crispus (Polygonaceae), y Jatropha dioica (Euphorbiaceae) se incorporan a tabletas cuyo consumo se mide diez días después y se cuentan las excretas producidas. $T$. glauca, $D$. bicolory $P$. pulchella reducen significativamente $(\mathrm{p}<0.01)$ la alimentación de $S$. granarius.

En el Instituto de Ciencias del Mar y Limnología de la UNAM se han realizado diversos estudios sobre ecología química marina, los cuales también tienen como objetivo realizar una búsqueda de metabolitos secundarios con utilidad potencial en la variada flora y fauna de los mares mexicanos. Se ha comprobado que los metabolitos de las esponjas marinas poseen propiedades antimicrobianas, antivirales, antineoplásicas, cardiotónicas y tóxicas; éstas nos han proporcionado una variedad de compuestos químicos que incluyen terpenoides, esteroides, carotenoides y compuestos fenólicos y nitrogenados, algunos con estructuras novedosas y/o con algún tipo de bioactividad. Por ejemplo, de una esponja mexicana, Haliclona sp., se aislaron e identificaron varios compuestos antimicrobianos derivados de la isoquinolina; de Aplysina (Verongia) triona, se aislaron cuatro nuevos metabolitos secundarios que se consideran derivados de la 3,5-dibromotirosina. También hay varios estudios sobre antimicrobianos de algas marinas y dinoflagelados (Cruz et al., 1989). En un estudio con los géneros Muricea, Lophogorgia y Pacifigorgia se encontraron sustancias que inhibieron el desarrollo de bacterias tales como Staphylococcus aureus, Escherichia coli y Bacillus subtilis, y mostraron discreta actividad contra la levadura Candida stellatoidea (Del Río-Estrada, 1989),

Ondarza (1989) menciona algunos aspectos sobre feromonas de Triatoma mazzottii, vector potencial de la enfermedad de Chagas. Se ha observado la presencia de una feromona sexual, producida en las glándulas abdominales de las hembras, y feromonas de agregación en heces de hembras adultas, ninfas y machos. Cibrián-Tovar (1989) reporta que la feromona sexual de Heliothis subflexa es una mezcla de aldehídos, acetatos y alcoholes los cuales son determinantes para iniciar el cortejo, localizar la fuente emisora de feromona y aterrizar en la fuente de estímulo (hembra o trampa).

Fabila (1989) describe algunos áspectos del comportamiento de los escarabajos del estiércol, Scarabaeinae. Los semioquímicos están asociados a una gran cantidad de pau- 
tas de comportamiento a nivel intraespecífico e interespecífico; en este último, son importantes los repelentes hacia competidores (mosca del género Calliphora) y los antibióticos con los que se impregna el alimento, los cuales evitan la proliferación de hongos que pueden, sin la presencia de los padres, matar a las larvas.

Reyes Chilpa (1989) realizó diversas pruebas de campo con veintidos maderas de la Selva Lacandona; el duramen de Astronium graveolens (Anacardiaceae), Manilkara zapota (Sapotaceae) y de las leguminosas Dialium guianense, Lonchocarpus castilloi y Lysiloma acapulcensis, es altamente resistente al biodeterioro, mientras que el de Platymiscium yucatanum (Leguminosae) es poco durable. Del extracto hexánico de $L$. castilloi se aislaron cinco nuevos flavonoides, los cuales fueron nombrados como castillenos A, B, C, D y $\mathrm{E}$, los cuales poseen el esqueleto de la 2-metoxi, 2-bencil furanocumara-nona.

En años recientes, los estudios de ecología química se han enriquecido con las contribuciones de EspinosaGarcía que junto con Langenheim (1991a,b,c) realizaron varias investigaciones sobre el efecto de algunos fenotipos de aceites esenciales de las hojas de Sequoia sempervirens sobre el desarrollo de diversos hongos con etapas endofíticas. Las especies probadas fueron Botrytis cinerea, Pestalotiopsis funerea, Phomopsisocculta, Seiridium juniperi, Cryptosporiosis abietina y Meria parkeri. Los cuatro fenotipos de aceites esenciales inhibieron de modo uniforme a algunas de estas especies y tuvieron un efecto diferencial sobre otras. La susceptibilidad a los cuatro fenotipos varió ampliamente, intraespecífica e interespecíficamente hablando. Los patógenos específicos de coníferas fueron los menos susceptibles al tratamiento con los aceites esenciales, mientras que el endofito de Sequoia fue el más susceptible. La diversidad de respuestas de todas las especies de hongos utilizadas en las pruebas, sugiere que los terpenoides de Sequoia pueden tener una importancia diferencial intra e interespecífica para prevenir la actividad patogénica de las especies que se encuentran en su follaje. Los autores proponen la hipótesis de que el endofitismo mutualístico probablemente evolucionó de patógenos latentes, puesto que muchos mutualistas endofíticos están estrechamente relacionados a patógenos virulentos; además, algunos otros endofitos pueden causar enfermedades cuando la planta se encuentra sometida a algún estrés. Dos de los componentes mayoritarios de la fracción monoterpénica del aceite esencial de las hojas de Sequoia son el sabineno y el g-terpineno; Espinosa y Langenheim proponen que estos monoterpenos pueden actuar aditiva 0 sinergísticamente sobre los hongos endofitos.

Actualmente, en otros países se realiza un gran esfuerzo para formar especialistas en ecología química trabajando en el control de plagas y enfermedades diversas, y en la búsqueda de nuevos productos útiles en la industria. En cambio, en México se desconoce o subestima lo que esta especialidad podría aportar en estos campos. El personal especializado es sumamente escaso, y más aún, los apoyos financieros y logísticos. Sin embargo, la necesidad de racionalizar e intensificar la producción de alimentos y materias primas, y de proteger y restaurar la naturaleza a la vez, nos obliga a considerar con gran interés a la ecología química y buscar la participación conjunta de la industria y las instituciones de investigación y enseñanza superior en su desarrollo.

\section{LITERATURA CITADA}

Altieri MA, Trujillo J. 1987. The agroecology of corn production in Tlaxcala, México. Human Ecology 15: 189-220.

Amo R S del, Anaya AL 1978. Effect of some sesquiterpenic lactones on the growth of certain secondary tropical species. J. Chem. Ecol. 4: 305-313.

Anaya AL. 1976. Consideraciones sobre el potencial alelopático de la vegetación secundaria. En: Gómez-Pompa A, Vázquez-Yanes C, Amo R S del, Butanda A, edrs. Investigaciones sobre la regeneración de selvas en Veracruz, México. CECSA, México, 428-445.

Anaya AL. 1989. Papel de los aleloquímicos en el manejo de los recursos naturales. Bol. Soc. Bot. México 49: 85-98.

Anaya AL, Rovalo M. 1976. Alelopatía en plantas superiores: diferencias entre el efecto de la presión osmótica y los alelopáticos sobre la germinación y el crecimiento de algunas especies de la vegetación secundaria de una zona cálido-húmeda de México. En: Gómez-Pompa A, Vázquez-Yanes C, Amo R S del, Butanda A, edrs. Investigaciones sobre la regeneración de selvas en Veracruz, México. CECSA, México, 388-427.

Anaya AL, Amo R S del. 1978. Allelopathic potential of Ambrosia cumanensis H.B.K. (Compositae) in a tropical zone of México. J. Chem. Ecol. 4: 289-304.

Anaya AL, Roy Ocotla G, Ortiz LM, Ramos L. 1982. Potencial alelopático de las principales plantas de un cafetal. En: Jiménez E, Gómez Pompa A, edrs. Estudios ecológicos en el agroecosistema cafetalero. Simposio del Instituto Nacional de Investigaciones sobre Recursos Bióticos. Xalapa, Ver. CECSA, México, 1-25.

Anaya AL, Ramos L,Hernández JG, Cruz-Ortega R.1987a. Allelopathy in Mexico. En: WallerGR, edr. Allelochemicals: role in agriculture and forestry. ACS Symposium Series 330. American Chemical Society, Washington, D.C., 89-101.

Anaya AL, Ramos L, Cruz-Ortega R, Hernández JG, Nava V. 1987b. Perspectives on allelopathy in Mexican traditional agroecosystems: a case study in Tlaxcala. J. Chem. Ecol. 13: 2083-2101.

Anaya, A.L, Gliessman SR, Cruz-Ortega R, Rosado-May F, y Nava V. 1988. Comparative effects of allelopathic weeds used as cover crop on the floristic potential of soils. En: Global perspectives on agroecology and sustainable agriculture. Proceedings of the Sixth International Scientific Conference of the International Federation of Organic Movements (IFOAM). University of California, Santa Cruz.

Anaya AL, Calera MR, Mata R, Pereda-Miranda R.1990. Allelopathic potential of compounds isolated from Ipomoea tricolor Cav. (Convolvulaceae). J. Chem. Ecol. 16: 2145-2152.

Anaya AL, Hernández-Bautista BE, Jiménez-Estrada M, VelascoIbarra L. 1992. Phenylacetic acid as a phytotoxic compound of corn pollen. J. Chem. Ecol. 18: 897-905.

Arízaga S, Ezcurra E. 1989. Efecto de la variabilidad foliar sobre el gremio de artrópodos epífitos del «Madroño» (Arbutusxalapensis H.B.K.) en Villa del Carbón, Edo. de México En: Resúmenes de la I Reunión Nacional de Ecología Química. Instituto de Fisiología Celular y Centro de Ecología. UNAM, México, 14. Brower LP. 1969. Ecological chemistry. Sci. Am. 220: 22-29. 
Brower LP, Brower JVZ. 1964. Birds, butterflies, and plant poisons: a study in ecological chemistry. Zoologica 49: 137-159.

Brower LP, Ryerson WN, Coppinger SC, Glazier SC. 1968. Ecological chemistry and the palability spectrum. Science 161: 13491351.

Calera MR, Soto F, Sánchez P, Bye R, Hernández-Bautista B, Anaya AL, Lotina-Henssen B, Mata R. Cytotoxic, antifungal and growth-inhibitory sesquiterpene lactones from Ratibida mexicana, and the inhibition of photosynthetic electron transport by isoalloalantolactone. Phytochem. (En prensa).

Calera MR, Anaya AL, Gavilanes-Ruíz M. 1995. Effect of phytotoxic resin glycoside on activity of $\mathrm{H}^{+}$-ATPase from plasma membrane. J. Chem. Ecol. 21: 289-297.

Castañeda P, García MR, Hernández-Bautista BE, Torres BA, Anaya AL, Mata R. 1992. Effects of some compounds isolated from Celaenodendron mexicanum Standl (Euphorbiaceae) on seeds and phytopathogenic fungi. J. Chem. Ecol. 18: 10251037.

Chacón JC, Gliessman SR. 1982. The use of the non weed concept in traditional tropical agroecosystems of Southeastern Mexico. Agroecosystems 8: 1-11.

Cibrian-Tovar J. 1989. Feromona de Heliothis subflexa (Guenée) (Lepidoptera: Noctuidae): Relación entre estructura química y comportamiento. En: Resúmenes de la I Reunión Nacional de Ecología Química. Instituto de Fisiología Celular y Centro de Ecología, UNAM, México, 16.

Collera-Zúñiga O. 1956. Estudio del aceite esencial de Piper auritum. Tesis de Licenciatura. Facultad de Química, UNAM, México.

Cruz F, Gómez P, Ríos T. 1989. Antibiosis y toxicidad en esponjas marinas. En: Resúmenes de la I Reunión Nacional de Ecología Química. Instituto de Fisiología Celular y Centro de Ecología, UNAM, México, 17.

Cruz-Ortega R, Anaya AL, Gavilanes-Ruíz M, Sánchez-Nieto S, Jiménez-Estrada M. 1990. Effect of piquerol on the $\mathrm{H}^{+}$ATPase activity of microsomes from Ipomoea purpurea, as a possible allelopathic mode of action. J. Chem. Ecol. 16: 22532261.

Dethier VG. 1954. Evolution of feeding preferences in phytophagous insects. Evolution 8: 33-54.

Dirzo R, Anaya AL. 1989. Introducción a la primera reunión de ecología química. Resúmenes de la I Reunión Nacional de Ecología Química. Instituto de Fisiología Celular y Centro de Ecología. UNAM, México, 9-10.

Ehrlich PR, Raven PH. 1965. Butterflies and plants: a study in coevolution. Evolution 18: 586-608.

Einhellig FA. 1989. Current status and future challenges in allelopathy. En: Report on the workshop on chemical interactions between organisms. International Foundation for Science-IFS. Santiago de Chile.

Espinosa-García FJ, Langenheim J. 1991a. Effects of sabinene and g-terpinene from coastal redwood leaves acting singly or in mixtures on the growth of some of their fungus endophytes. Biochem. System. Ecol. 19: 643-650.

Espinosa-García FJ, Langenheim J. 1991b. Effect of some leaf essential oil phenotypes in coastal redwood on the growth of several fungi with endophytic stages. Biochem. System. Ecol. 19: 629-642.

Espinosa-García FJ, Langenheim J. 1991c. Effect of some essential oil phenotypes from coastal redwood on grow th of its predominant endophytic fungus, Pleuropaconema sp. J. Chem. Ecol. 17: 1837-1857.

Fabila ME. 1989. El papel de los semiquímicos en el comportamiento reproductor de algunos escarabajos del estiercol (Scarabaeidea,
Scarabaeinae). En: Resúmenes de la I Reunión Nacional de Ecología Química. Instituto de Fisiología Celular y Centro de Ecología, UNAM, México, 21.

Fraenkel GS. 1959. The raison d'être of secondary plant substances. Science 129: 1466-1470.

González de la Parra M, Anaya AL, Espinosa FJ, Jiménez M, Castillo R. 1981. Allelopathic potential of Piqueria trinervia (Compositae) and piquerols A and B. J. Chem. Ecol. 7: 509515.

González de la Parra M, Chávez-Peña D, Jiménez-Estrada M, Ramos-Mundo C. 1991. Acaricidal potential of piquerols A and B against Boophilus microplus. Pestic. Sci. 33: 73-80.

Graue WB, Rovalo M. 1982. Potencial alelopático y microbicida de Helieta parvifolia. Biotica 7: 405-416.

Harborne JB. 1989. Introduction to ecological biochemistry. Academic Press, New York.

Jiménez-Sierra C, Dirzo R. 1989. Preferencias alimenticias de Melanonotus globosus (Tettigoniidae): Un enfoque experimental. En: Resúmenes de la I Reunión Nacional de Ecología Química. Instituto de Fisiología Celular y Centro de Ecología. UNAM, México, 24.

Müller CH. 1970. Phytotoxins as plant habitat variables. Rec. Adv. Phytochem. 3: 105-127.

Nava-Rodríguez V, Amo R S del, Anaya AL. 1985. Efecto de aceites esenciales de estados juveniles de especies primarias, contra el ataque de hongos, como un mecanismo de defensa. En: Gómez Pompa A, Amo R S del, edrs. 1985. Investigaciones sobre la regeneración de selvas altas en Veracruz, México II. Alhambra Mexicana, México, 283-298.

Ondarza RN. 1989. Feromonas, conducta y ecología de Triatomineos: vectores de la enfermedad de Chagas. En: Resúmenes de la I Reunión Nacional de Ecología Química. Instituto de Fisiología Celular y Centro de Ecología. UNAM, México, 26.

Pereda-Miranda R, Mata R, Anaya AL, Wickramaratne DB, Pezzuto JM, Kinghorn AD. 1993. Tricolorin A, major phytogrowth inhibitor from Ipomoea tricolor. J. Natural Products 56:571582.

Ramos-Prado JM. 1981. Interacciones químicas entre especies primarias y secundarias de selva alta perennifolia. Tesis de Licenciatura, Facultad de Ciencias, UNAM.

Reyes-Chilpa R. 1989. Defensas químicas de la madera a los descomponedores. En: Resúmenes de la I Reunión Nacional de Ecología Química. Instituto de Fisiología Celular y Centro de Ecología. UNAM, México, 28.

Río-Estrada C del. 1989. Papel bioquímico de los celenterados marinos en la ecología microbiana. En: Resúmenes de la I Reunión Nacional de Ecología Química. Instituto de Fisiología Celular y Centro de Ecología. UNAM, México, 29.

Rodríguez-Hernández C, Lagunas-Tejeda A. 1989. Actividad insecticida de polvos y extractos acuosos vegetales. En: Resúmenes de la I Reunión Nacional de Ecología Química. Instituto de Fisiología Celular y Centro de Ecología. UNAM, México, 30.

Rosado-May FJ, García RE. 1985. Incidencia de la «mustia hilachoza» (Tanatephorus cucumeris) en frijol común como resultado del manejo del suelo. Rev. Mex. Fitopatol. (México) 3: 92-99.

Rosado-May FJ, Gliessman SR, Alejos MP. 1986. El «cadillo» (Bidens pilosa), arvense asociada al maíz en la Chontalpa Tabasco: Planta con propiedades nematicidas. Rev. Mex. Fitopatol. (México) 4: 124-132.

Rovalo M. 1973. Detección de inhibidores de la germinación y el crecimiento en las hojas de Piper hispidum. Tesis de Licenciatura. Facultad de Ciencias. UNAM.

Rovalo M. 1978. Bioensayos con la «gobernadora» (Larrea divaricata 
Cav.) como posible planta alelopática y/o herbicida. Primera Reunión sobre los Reguladores de las Plantas y los Insectos, Consejo Nacional de Ciencia y Tecnología, Monterrey, México.

Uribe S, Alvarez R, Peña A. 1984. Effects of $B$ - pinene, a nonsubstituted monoterpene, on rat liver mitochondria. Pest. Bioch. Physiol. 22: 43-50.

Uribe S, Ramírez J, Peña A. 1985. Effects of B- pinene on yeast membranes functions. J. Bacteriol. 161: 1195- 1200.

Villavicencio MA, Juárez-Monroy A. 1989. Un bioensayo para la exploración de extractos de plantas como antialimentarios de Sitophilus granarius L (Coleoptera: Curculionidae). En: Resúmenes de la I Reunión Nacional de Ecología Química. Instituto de Fisiología Celular y Centro de Ecología, UNAM, México, 46.
Waller GR, Kumari O, Friedman J, Friedman N, Chou CH. 1986. Caffeine autotoxicity in Coffea arabica L. En: Putnam AR, Tang CS, edrs. The science of allelopathy. NewYork: John Wiley and Sons, 243-269.

Waller GR, Anaya AL, Sagrero-Nieves L, Celis Ochoa Cordero AB, Kumari D, Friedman J, Friedman N, Chou CH. 1989. A problem in coffee plantations: autotoxicity of caffeine and other compounds. ASIC. Proceedings of $13^{\circ}$ Colloque. Paipa, Colombia, 363-371.

Zavaleta-Mejía E. 1989.Effect of volatiles emanated from Brassicaceous (Cruciferous) residues on some soil-borne plant pathogens. En: International Foundation for Science (IFS). Report on the workshop on chemical interactions between organisms. Santiago, Chile, 118-123. 